\title{
Evaluation outcome of the regional approach of physiotherapy, conventional approach of physiotherapy and non-steroidal anti-inflammatory drugs (NSAID) on anxiety for chronic mechanical spinal pain disorder
}

\begin{abstract}
Introduction: Chronic pain and psychiatric disorders frequently co-occur. Anxiety followed by chronic spinal pain is not properly addressed. The purpose of the study is to compare effectiveness of the regional approach (new approach) of physiotherapy treatment, NSAID and conventional physiotherapy approach on pain and disability status for chronic mechanical spinal pain disorder.
\end{abstract}

\begin{abstract}
Materials and Method: A repeated measurement ANOVA clinical trial design was carried out to test the hypothesis. 104 patients between 20-65 years old with chronic mechanical spinal pain were randomly assigned into 3 different groups. Measurements were taken before starting of intervention, middle of the intervention and at the end of the intervention. The regional group (30 patients) received 8 sessions of regional approach of Physiotherapy, conventional group (35 patients) received 8 sessions of conventional approach of physiotherapy treatment and drug group ( 39 patients) received 21 to 30 days NSAID as per prescription of an orthopedic Professor. To eliminate bias, clinical physiotherapists were also randomly allocated into either for regional approach physiotherapy group or as usual physiotherapy group. A treatment protocol of regional approach was developed and handed over to clinical physiotherapists for regional approach group to deliver treatment. Outcome was monitored by Hamilton Anxiety rating scale.
\end{abstract}

Results: The results of the study show that the regional approach of physiotherapy treatment, NSAID and conventional physiotherapy approach are differentially effective on anxiety status $(\mathrm{P}<0.05)$. The outcome of anxiety was differently effective over different occasions of treatment (pretest, mid-test and posttest) for chronic mechanical spinal pain $(\mathrm{P}<0.05)$. Alongside the effect of time and treatment interaction of anxiety was also found to be statistically significant $(\mathrm{P}<0.05)$. Greater improvement of anxiety has been shown in regional group in comparison to conventional and NSAID group.

Conclusion: Regional approach of treatment has been found to be a new evidence of treatment for chronic mechanical spinal pain in reduction of anxiety level. The study demonstrates short term outcome of the treatment methods. However, a further study is required to see the long term effect of those treatments.

Keywords: regional approach of physiotherapy, conventional approach of physiotherapy, non-steroidal anti-inflammatory drugs (NSAID), chronic mechanical spinal pain disorder
Volume 3 Issue 4 - 2018

\author{
Nasirul Islam,' M Mazibar Rahman, ${ }^{2}$ \\ Mohammad Habibur Rahman ${ }^{3}$ \\ 'Assoicate Professor and Vice Principal, Bangladesh Health \\ Professions Institute, Bangladesh \\ 2Professor, Department of Statistics, Jahangirmagar University, \\ Bangladesh \\ ${ }^{3}$ Associate Professor, Department of Physiotherapy, Bangladesh \\ Health Professions Institute, Bangladesh
}

Correspondence: Nasirul Islam, Associate Professor and Vice Principal, Bangladesh Health Professions Institute (BHPI), CRP, Dhaka, Bangladesh, Email nasirulpt@gmail.com

Received: June 22, 2018 | Published: July 05, 2018

\section{Introduction}

Spinal pain is an extremely common symptom in general population across the world. Neck pain (NP) and low back pain (LBP) are seemed to be massive public health problems because of multiple aspects including their high incidence, long duration, and social and economic impact.

According to a global review, the one-year prevalence of neck pain ranges between $16.7 \%$ and $75.1 \%$ (mean 37.2\%). ${ }^{2}$ Another study shows that the overall occurrence of neck pain in the general population is estimated between $0.4 \%$ and $86.8 \%$ and one-year prevalence ranges from $4.8 \%$ to $79.5 \%$.

The prevalence of neck pain has been growing up over last 2 decades $^{4}$ and is now second to back pain, the most common musculoskeletal disorder. ${ }^{5}$ More than half of all adults are found to be experienced neck pain during the past 6 months, and women are particularly more vulnerable than men to develop and suffer from persistent neck pain. ${ }^{6}$ One study ${ }^{7}$ found a significant positive associations between chronic pain and individual with anxiety disorders and odds ratios $(\mathrm{OR}=1.92$ to 4.27$)$. However, the strongest associations were observed with panic disorder $(\mathrm{OR}=4.27)$ and posttraumatic stress disorder $(\mathrm{OR}=3.69)$.

Chronic pain is persistent long-term pain, sometimes lasting throughout life. Even chronic pain may present with acute episodes of pain and persists for 12 weeks or more. ${ }^{8,9}$ Transduction, transmission, modulation and perception are the steps of feeling of pain. Perception is the processing and recognition of the pain in the brain, the thalamus transmits signals to the somato-sensory cortex (sensation), frontal cortex (thinking) and limbic system (emotional response). In fact, suffering from chronic pain has dramatic impacts 
on the lives of an individual and create a complex problem since it can lead to depression, anxiety (fear or anticipation of future pain), and immobilization, all of which may in turn increase pain. ${ }^{10} \mathrm{Chronic}$ pain conditions are extremely diverse and can affect peripheral, spinal, central, and psychological levels. The mechanisms of these are not fully understood. On the other hand, chronic pain patients generally have a poorer prognosis due to psychological factors. ${ }^{11}$ Health care costs and productivity losses most often associated with chronicity and may be excess 50 billion US Dollar annually in US. ${ }^{12}$ Alongside the prevalance of major depression in Chronic lower back pain is 3 to 4 times higher comparing general population.

Psychosocial model of chronic pain explains the relationship between pain, disability and muscle spasm with mal-adaptive coping strategies including negative thinking, pathological fear and abnormal anxiety regarding pain, avoidant behavior, catastrophizing and hypervigilance. ${ }^{13}$ Social factors such as the compensation system, work place disputes, work and family tensions and cultural issues affecting beliefs reinforce the psychological factors that can increase the central drive of pain. ${ }^{14}$ In contrast positive factors such as adaptive coping strategies, appropriate pacing and distraction can have a descending inhibitory effect on pain via the forebrain which means positive factors reinforce in reduction of pain perception. ${ }^{15}$

Chronic pain may origin from sources that are extrinsic to the nervous system (for example, ongoing injury or inflammation), but it can also be intrinsic and the result of abnormal hypersensitivity in neuropathic or partially denervated structures. Neuropathic pain generally influences the musculoskeletal system, and a crucial component of this type of pain is muscle spasm or shortening. Spasm can cause pain localized to muscle, but sustained muscle spasm or shortening mechanically overburden tendons and their attachments, and can initiate pain in these structures. Thus sustain muscle spasm can cause pain across muscle and tendon. At the same time shortened muscle can increase joint pressure, upset alignment and cause arthralgia. For example, for spinal case, shortening in para-spinal muscle can compress a disc space and narrow intervertebral foramina. The nerve root may then irritate through pressure of bulging disc. A vicious cycle is developed which ultimately produce neuropathy.

Despite of temporal relationships between pain conditions and depression the temporal relationships between pain conditions and anxiety disorders remains largely ignored. ${ }^{7}$ Several studies found the effectiveness of exercise on depression but from the best knowledge of the researcher no study carried out showing the efficacy of regional approach of physiotherapy on depression for chronic mechanical spinal pain disorder.

\section{Materials and methods}

Following mechanical diagnosis and therapy approach a total 104 patient with chronic mechanical spinal pain disorder were included in the study (30 in RAP group, 35 in CAP group and 39 in NSAID group). One study ${ }^{16}$ found that each arm minimum sample size was 28 that would give $5 \%$ significant and $80 \%$ power of the study for this repeated measures design. Hamilton Anxiety rating scale was used to measure the outcome of the investigation.

\section{Statistical methods}

A repeated-measure ANOVA demonstrates the outcome change with alternative treatments over time. When the measurements are taken more than two times repeatedly over a period of time on the same dependent variable repeated measure ANOVA should be used. ${ }^{17}$
Repeated measure design was carried out in three different subject's groups (Regional, conventional and NSAID) and were observed several times (Pretest, mid-test and posttest). The subjects were observed at successive occasions to see how the treatment effects on outcome. Treatment providers (clinical physiotherapists) were not involved in data collection. Physiotherapists from RAP group and CAP groups were also randomized so that the skills and experiences don't influence outcome (Figure 1).

\section{Treatment methods}

\section{Conventional physiotherapy approach}

There is lack of published data showing different types of conventional treatments that Physiotherapists apply globally or locally for anxiety followed by chronic pain. However observational data reveals that conventional Physiotherapy for low back pain patients include Mckenzie approach, spinal mobilization, back muscles strengthening exercise, electrotherapeutic modalities including IRR and TENS, pelvic floor strengthening exercise, home exercise, Cyriax manipulation, Maitland mobilization, Mulligan mobilization, Neural mobilization and exercise therapy including leg muscle strengthening. Similarly, the conventional approach for neck pain patients are recognized as multimodal treatments such as McKenzie exercises in combination with manual therapy, exercise therapy, superficial and deep heating agents as well as traction. While several researches show the outcome of conventional physiotherapy approach on pain and disability, the outcome of anxiety was not addressed. Most of the conventional approaches of Physiotherapy are found to be merely local. Treatments integrating peripheral joint or muscles are rarely applied.

\section{Regional approach of physiotherapy treatment}

There is no simple, defined, time effective and robust effective physiotherapy interventional package for the chronic mechanical spinal pain. Therefore, the researcher developed a new protocol of physiotherapy treatment called the regional approach. The regional approach includes treatment addressing adjacent structures (muscles or joint). The literature reviews show that the chronic pain is multi factorial and this affect peripheral structure due to secondary changes or plastic changes of the nervous system. Myo-fascial release, stretching and strengthening targeting adjacent joint and muscles are fundamental components of regional approach. The basic different of regional approach from conventional one is, regional approach covers a whole area. For example, for neck pain, regional approach covers muscles and joints pectoral girdle and upper thoracic spine along with neck. In contrast for lower back pain, regional approach covers joints and muscles of pelvic girdle.

The regional approach evaluates mal orientation of joints and muscles and correct those with active/manual therapy illustrated in the protocol. The concept of the treatment is to create window by adjusting peripheral components so that local impact can be eased. The approach is thought to be effective in reduction of pain and disability at a greater extend than conventional approach which ultimately will create impact on anxiety reduction.

\section{Protocol of regional approach}

1. Subjective assessment: personal history, past medical history, drug history

2. Objective assessment:

i. Observation: Local/regional asymmetrical to be noted. 
ii. Palpation note (local, neck/thorax/back): tenderness/muscle spasm/hypertrophied/atrophied muscles and their distribution

iii. Palpation note (regional, shoulder, scapula, pectoralis for neck and buttock, iliotibial band, Sacroiliac, piriformis, hamstring and leg): tenderness/muscle spasm/hypertrophied/tightness of adjacent peripheral muscles/atrophied muscles/trigger point/ adhesions and their distribution Analysis of movement: Hyper mobile/hypo mobile/ local limitation/ regional limitation/ evaluation of shoulder/hip joint

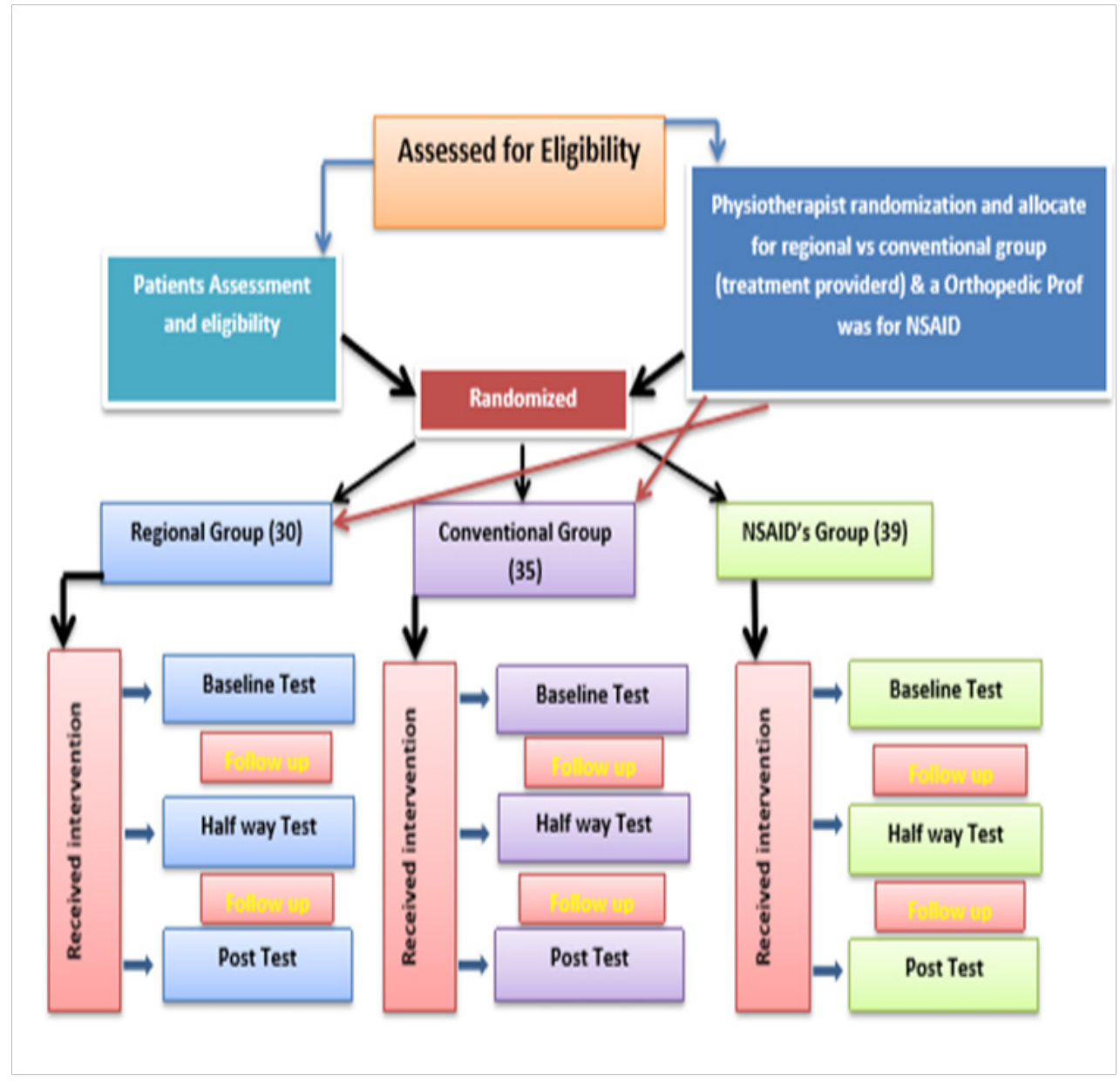

Figure I: Experimental structure.

\section{Treatment concept for regional approach (Figure 2)}

Back exercise includes: Multifidus muscle stretching/strenthening, Quadratus lumborum stretching, Quadriceps muscle stretching, Hamstring stretching, Erector spine and Letismus Dorsi muscles stretching, Piriformis muscle stretching, Ilitibial band strethcing, Drilling stretching of hip rotators and Myfacial release.

For neck: Manual release of pectoralis (muscles and tendon), Trapezius muscles stretching, Splenius Muscles stretching, Scalene Muscles stretching, Sternocleido mastoid Muscle, Suboccipital muscle stretching, Rhomboid Muscles stretching, Driling stretching for shoulder rotators.

Strengthening weak muscles as per requirement of the patients in the form of isometric/isotonic/concentric/eccentric (dosages are given in protocol). Manual therapy includes mobilization of thoracic spine for both cervical and lower back disorders.

\section{Non-steroidal Anti-inflammatory Drugs (NSAIDs)}

NSAIDs are a group of drugs for pain, fever and inflammation and frequently prescribed drugs for chronic spinal pain disorder. NSAID has an effect of pain reduction but has side effect simultaneously. Gastritis and renal failure are very common observed side effect which can create negative impact on anxiety level.

An Orthopedic Professor prescribed this drug for 39 chronic Mechanical spinal pain disorder patients. Data collector took written permission from the eligible patients.Data collector maintained follow up with all listed patients under the study. Photocopy of each prescription was taken with the verbal consent from patients and Doctor. Tablet tenoxicam $20 \mathrm{mg}$ was the most common NSAID that orthopedic consultant used for the patients under this study. In addition, another $\mathrm{H} 2$ blocker was given along with NSAID to prevent side effect associated with NSAID's intake. The dosage of the drug is once a day $(20 \mathrm{mg})$. However, the patients under this group used drugs from 21 to 30 days. It is because, chronicity of the problem. 


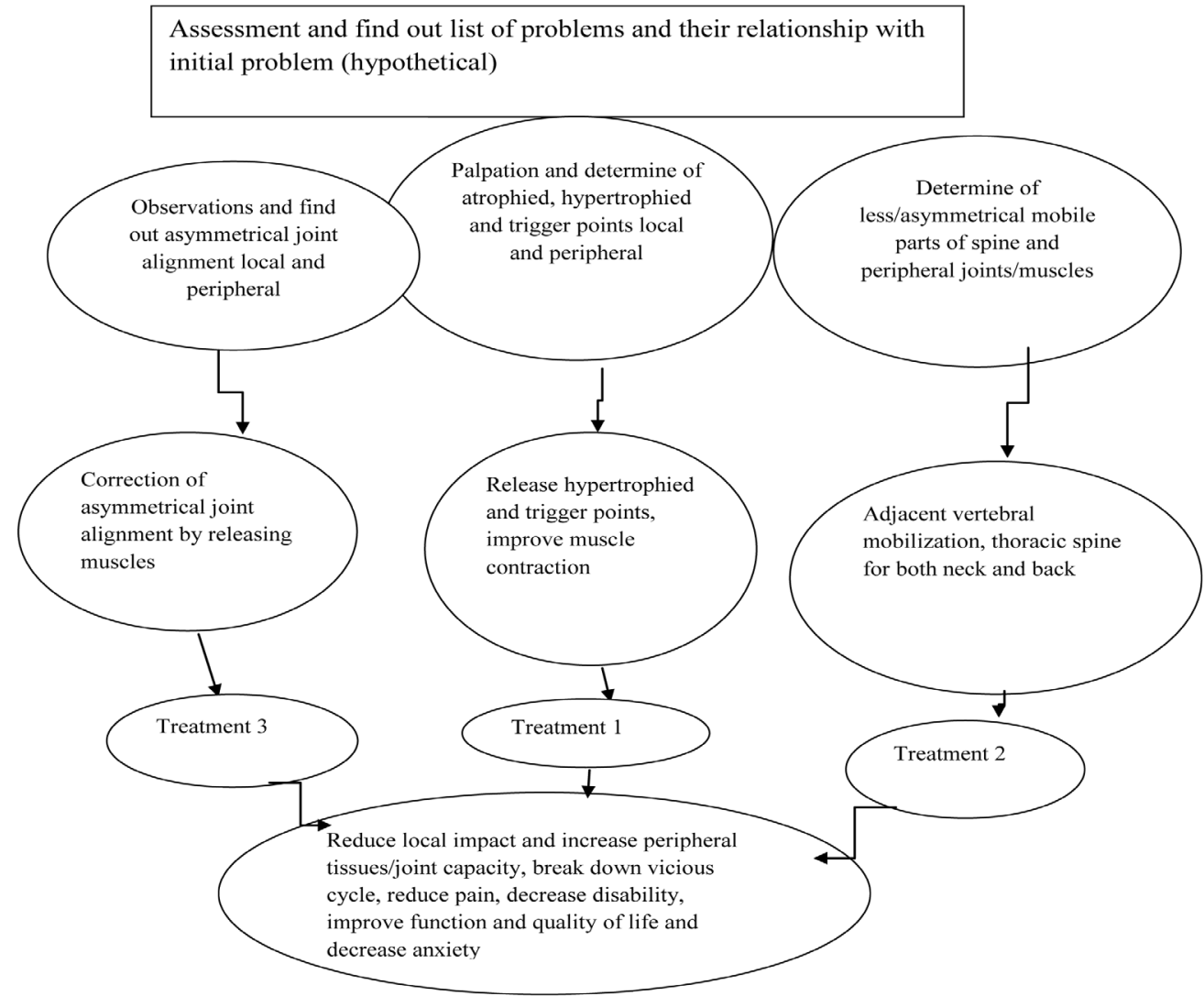

Figure 2: Treatment model of regional approach (intervention by therapist + home exercise).

A systematic review results shows that the effect of NSAIDs is significantly different in relation to placebo for back pain disorder. But qualitative analysis showed that there is conflicting evidence (level 3) that NSAIDs are more effective than paracetamol for acute low back pain and moderate evidence (level 2) that NSAIDs are not more effective that other drugs for acute low back pain and there is strong evidence (level 1) that various types of NSAIDs are equally effective for acute low back pain. ${ }^{18} \mathrm{~A}$ few studies suggest that tricyclic antidepressants and cognitive behavioral therapy may be effective for depression. But the effect of NSAID on anxiety level as per researchers concern is so far not investigated.

\section{Inclusion criterion}

Persistent cervical or lumber pain for more than 3 months, pain of mechanical origin, radiating or local pain, age group up to 65 years and patients were willing to receive minimum 8 sessions of treatment under physiotherapy group and complete course of NSAID therapy.

\section{Exclusion criteria}

Pathological source of pain including TB spine, spinal tumor, cervical and back problem presented simultaneously, abscess, early spinal fracture, red flags syndrome and patients were excluded in case of having any double treatment exposure.

\section{Data collection tools and measurement}

Hamilton Anxiety rating scale (The HAM-A) is a clinician-based questionnaire to determine clinical anxiety level. The scale is made of 14 symptoms including anxious mood, tension, fears, insomnia, intellectual, depressed mood, somatic (muscular), somatic (sensory), cardiovascular symptoms, respiratory symptoms, gastrointestinal symptoms, genitourinary symptoms, autonomic symptoms, behavior at interview. The scale also defined characteristics under each symptom. For example intellectual component covers difficulty in concentration and poor memory. Each item is scored on a basic numeric scoring of 0 (not present) to 4 (severe). However, one study ${ }^{19}$ tested the reliability and validity of the scale and concluded that the reliability and concurrent validity of the HAM-A and its subscales were sufficient, despite the scale has limited usage in the evaluation of anxiolytics.

\section{Participants}

104 patients with chronic mechanical spinal pain were randomly assigned into three groups. 30 patients were assigned in regional, 35 in conventional group and 39 in NSAIDs group. Physiotherapists having similar qualification, skills and experience are also randomly assigned in regional and conventional group. A base line assessment 
was taken before the starting of the treatment. A half way evaluation was done at the middle of the treatment for regional, conventional group and NSAID group. Finally, $3^{\text {rd }}$ measurement was taken at the end of the treatment.

\section{Data Analysis}

SPSS version 25, Genstat version 5 and Microsoft excel were used to analyze data. Hamilton anxiety rating scale: Sum score of all domain score $/ 56 * 100=\%$ of score

\section{Results}

The demographic results of the study show that out of 30 participants in regional group, $n=18(60 \%)$ were male and $n=12$ $(40 \%)$ were female. Whereas in conventional group out of 35 patients, $\mathrm{n}=16(46 \%)$ were male and $\mathrm{n}=19(54 \%)$ were female and in NSAID group out of 39 patients, $\mathrm{n}=16(41 \%)$ were male in comparison to $\mathrm{n}=23(59 \%)$ female. Overall out of 104 participants $\mathrm{n}=50(48 \%)$ were male and $n=54(52 \%)$ were female. The mean age of the participants of the both regional group and conventional group was 40 years in comparison to 37 years in NSAID group. The mean duration of suffering from pain is respectively 30 months, 35 months and 30 months for regional, conventional and NSAID group (Table 1).

Table I Demographic information of the participants

\begin{tabular}{llll}
\hline & $\begin{array}{l}\text { Regional } \\
\text { group } \\
\text { N=30 }\end{array}$ & $\begin{array}{l}\text { Conventional } \\
\text { group } \\
\mathbf{N}=\mathbf{3 5}\end{array}$ & $\begin{array}{l}\text { NSAID } \\
\text { group } \\
\mathbf{N}=39\end{array}$ \\
\hline Sex Male $\quad$ Female & $\mathrm{N}=18(60 \%)$ & $\mathrm{N}=16(46)$ & $\mathrm{N}=16(41 \%)$ \\
Age Range in years & $\mathrm{N}=12(40 \%)$ & $\mathrm{N}=19(54 \%)$ & $\mathrm{N}=23(59 \%)$ \\
$\begin{array}{l}\text { Mean suffering from } \\
\text { pain in month }\end{array}$ & 30 & $21-65$ & $22-58$ \\
\hline
\end{tabular}

Table 2 shows mean effect of anxiety level among 3 treatment groups in three different occasions. The mean anxiety reduction in regional, conventional respectively 13.34 and 18.16 unit but 1.91 units increased in NSAID group.

Table 2 Descriptive Statistics of within group mean anxiety score

\begin{tabular}{lllll}
\hline \multicolumn{2}{l}{ Descriptive statistics anxiety } & & & \\
\hline \multicolumn{2}{l}{ Dependent variable: outcome } & & & N \\
\hline Treatment & Time & Mean & Std. deviation & 35 \\
\hline \multirow{4}{*}{ Conventional } & Pretest & 50.71 & 17.642 & 35 \\
& Midway test & 34.9 & 14.458 & 35 \\
& Posttest & 32.55 & 16.999 & 105 \\
& Grand Total & 39.39 & 18.173 & 39 \\
NSAIDs & Pretest & 27.76 & 16.321 & 39 \\
& Midway test & 39.79 & 14.285 & 39 \\
& Posttest & 29.67 & 17.638 & 117 \\
& Grand Total & 32.41 & 16.856 & 30 \\
Regional & Pretest & 26.84 & 8.922 & 30 \\
& Midway test & 18.21 & 6.797 & 30 \\
& Posttest & 13.5 & 6.541 & 90 \\
\hline
\end{tabular}

Table 3 The results of the repeated measure ANOVA showed that there was a significant main effect of the type of treatment (Regional, conventional and NSAID) on the effectiveness of anxiety, $\mathrm{F}=56.61$, $\mathrm{P}<0.01$. There was a significant main effect of time (Pretest, midway test and post intervention) on the effectiveness of anxiety, $\mathrm{F}=14.51$, $\mathrm{P}<0.01$. There was also a significant interaction between the types of treatment and time on the effectiveness of anxiety, $\mathrm{F}=10.11, \mathrm{P}<0.01$.

Table 3 Analysis of Variance for Anxiety (ANOVA)

\begin{tabular}{llllll}
\hline SV & DF & SS & MSS & F & Sig \\
\hline Time & 2 & 4832.82 & $2416.4 I I$ & 14.517 & $.000^{* *}$ \\
Error (a) & 101 & 23398.9 & 231.672 & & \\
Treatment & 2 & 18847.3 & 9423.644 & 56.616 & $.000 * *$ \\
Time*Treatment & 4 & 6735.62 & 1683.906 & 10.117 & $.000 * *$ \\
Error (b) & 202 & 43443.2 & 215.066 & & \\
Total & 311 & 73859 & 237.489 & & \\
\hline
\end{tabular}

Note '**'denote the significant different at I\% level and '*'denote the significant different at $5 \%$ level

Table 4 the post hoc LSD tests for multiple comparison showed that the mean effect of anxiety in three pairs of times (Pretest-posttest, pretest-mid test and midtest- posttest) are found to be statistically significant $\mathrm{P}<0.05$.

Table 4 Multiple Comparisons of means anxiety of different pairs of time

\begin{tabular}{|c|c|c|c|c|}
\hline & (I) Time & (J) Time & Mean difference (I-J) & Sig. \\
\hline \multirow{6}{*}{ LSD } & \multirow{2}{*}{ Pretest } & Midtest & $3.99 *$ & $.039 *$ \\
\hline & & Post test & $10.28 *$ & $.000 * *$ \\
\hline & \multirow{2}{*}{ Midtest } & Pretest & $-3.99 *$ & $.039 *$ \\
\hline & & Post test & $6.29 *$ & $.001 * *$ \\
\hline & \multirow{2}{*}{ Posttest } & Pretest & $-10.28 *$ & $.000 * *$ \\
\hline & & Midtest & $-6.29 *$ & $.001 * *$ \\
\hline
\end{tabular}

Note **.The mean difference is significant at the .01 level.

Table 5 the mean effect of anxiety in all three pairs of treatment (Regional and Conventional, Regional and NSAID and Conventional and NSAID) are found to be statistically significant $\mathrm{P}<0.05$.

Table 5 Multiple Comparisons of means anxiety of different pairs of treatment

\begin{tabular}{|c|c|c|c|c|}
\hline & (I) Treatment & (J) Treatment & $\begin{array}{l}\text { Mean difference } \\
(I-J)\end{array}$ & Sig. \\
\hline \multirow{6}{*}{ LSD } & \multirow{2}{*}{ Regional } & Conventional & $-20.44^{*}$ & 0 \\
\hline & & NSAIDs & $-9.42 *$ & 0 \\
\hline & \multirow{2}{*}{ Conventional } & Regional & $20.44 *$ & 0 \\
\hline & & NSAIDs & $11.02 *$ & 0 \\
\hline & \multirow{2}{*}{ NSAIDs } & Regional & $9.42^{*}$ & 0 \\
\hline & & Conventional & $-11.02 *$ & 0 \\
\hline
\end{tabular}

Note **.The mean difference is significant at the .01 level.

Table 6 showed that the mean effect of anxiety in pretest-NSAID, posttest-NSAID are same. The effects of mid test-NSAID, PosttestConventional are same whereas other time and treatment interaction are found to be different.

Citation: Islam N, Rahman MM, Rahman MH. Evaluation outcome of the regional approach of physiotherapy, conventional approach of physiotherapy and nonsteroidal anti-inflammatory drugs (NSAID) on anxiety for chronic mechanical spinal pain disorder. Int Phys Med Rehab J. 20I8;3(4):279-285. DOI: $10.15406 /$ ipmrj.2018.03.00116 
Table 6 Multiple comparison test of anxiety (Time*Treatment)

\begin{tabular}{lll}
\hline Time & Treatment & Mean \\
\hline \multirow{2}{*}{ Pretest } & Regional & $27.000(\mathrm{~d})$ \\
& Conventional & $5 \mathrm{I} .200(\mathrm{a})$ \\
& NSAIDs & $25.033(\mathrm{e})$ \\
& Regional & $18.433(\mathrm{f})$ \\
Midtest & Conventional & $35.133(\mathrm{c})$ \\
& NSAIDs & $37.700(\mathrm{~b})$ \\
& Regional & $13.667(\mathrm{~g})$ \\
Posttest & Conventional & $34.100(\mathrm{~b})$ \\
& NSAIDs & $24.633(\mathrm{e})$ \\
SE & & 2.36 \\
LSD (5\%) & & 6.042 \\
\hline
\end{tabular}

Figure 3 The line diagram shows the changes of anxiety level among regional, conventional and NSAID groups during pretest, mid test and posttest. Overall, the regional group and conventional group show downward trend throughout the period although anxiety score increased in NSAID group. During pretest the mean score of anxiety in the regional group was just above 25 unit, which steadily reduced to just below 20 unit at the middle and further dropped to about 5 unit at the post test. While in conventional group, pretest score was just above 50 unit and dramatically decreased to about 35 unit during mid-test and remained same during posttest. In contrast, pretest score in NSAID group was about 25 unit which sharply increased above 35 during mid-test but further sharply decreased just above 25 unit. The regional group showed more improvement by $41 \%$ in relation to conventional group $36 \%$. But the NSAID groups shows to be increased anxiety by $7 \%$.

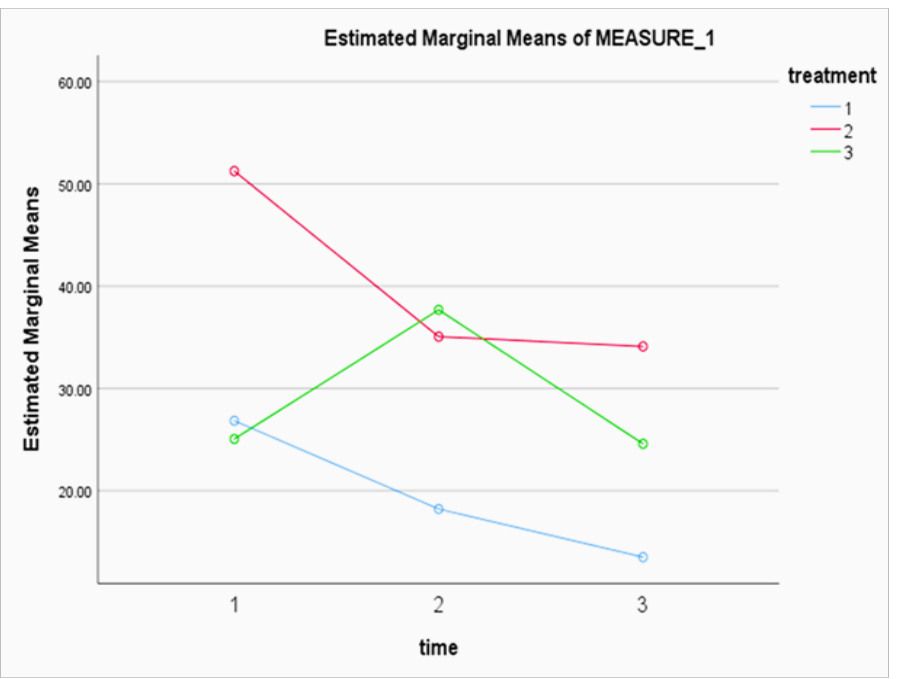

Figure 3 Line diagram showing the effect of Regional, Conventional and NSAID treatment on anxiety on different occasions of time.

**Note- $1=$ Regional group; 2 = Conventional group; 3 = NSAIDs group

\section{Discussion}

The results of the repeated measure ANOVA showed that there was a significant main effect of the type of treatment (Regional, conventional and NSAID) on the effectiveness of anxiety, $\mathrm{F}=56.61$,
$\mathrm{P}<0.01$. There was a significant main effect of time (Pretest, midway test and post intervention) on the effectiveness of anxiety, $\mathrm{F}=14.51$, $\mathrm{P}<0.01$. There was also a significant interaction between the types of treatment and time on the effectiveness of anxiety, $\mathrm{F}=10.11, \mathrm{P}<0.01$.

The post hoc LSD tests for multiple comparison showed that the mean effect of anxiety in three pairs of times (Pretest and posttest, Pretest-mid test and mid test and posttest) are found to be statistically significant $\mathrm{P}<0.05$. At the same time the mean effect of anxiety in all three pairs of treatment (Regional and Conventional, Regional and NSAID and Conventional and NSAID) are found to be statistically significant $\mathrm{P}<0.05$.

As per multiple comparison, time and treatment interaction, it showed that the mean effect of anxiety in pretest-NSAID, posttest-NSAID is same. The effect of mid test - NSAID, PosttestConventional is same whereas other time and treatment interaction are found to be different.

Several studies of exercise have reported improvements in depression scores after treatment. ${ }^{20,21}$ In these studies, improvement was reported in depression score that may be related to improvement in mood due to functional improvement.

Overall, the literature suggests that exercise treatment is more effective in treating depression than no treatment, and as effective as psychotherapy and antidepressant medication for patients with back pain. ${ }^{21}$

\section{Conclusion}

Overall the study was carried out to test the hypothesis which is regional approach of physiotherapy treatment; NSAID's and conventional physiotherapy approach are differentially effective on anxiety for chronic mechanical spinal pain disorder. The second hypothesis was the outcome of anxiety is differently effective over different occasions of treatment (pretest, mid-test and posttest) for chronic mechanical spinal pain.

The result of the study accepts hypothesis that regional approach of physiotherapy treatment, NSAID's and conventional physiotherapy approach are differentially effective on anxiety for chronic mechanical spinal pain disorder. The study result also accepts the second hypothesis that the outcome of anxiety is differently effective over different occasions of treatment (pretest, mid-test and posttest) for chronic mechanical spinal pain disorder.

The study demonstrates short term outcome of the treatment methods. However, a further study is required to see the long term effect of those treatments.

\section{Acknowledgments}

The authors express gratitude to the participants of the study. The authors thanks to Dr. Ismail Hossain for his kind support in statistically analysis and Saifuddin Ahmed Parvez for his contribution in data collection of the study.

\section{Conflict of interest}

The authors declare no conflict of interest.

\section{References}

1. Vos T, Barber RM, Bell B, et al. Global, regional, and national incidence, prevalence, and years lived with disability for 301 acute and chronic diseases and injuries in 188 countries, 1990-2013: a systematic

Citation: Islam N, Rahman MM, Rahman MH. Evaluation outcome of the regional approach of physiotherapy, conventional approach of physiotherapy and nonsteroidal anti-inflammatory drugs (NSAID) on anxiety for chronic mechanical spinal pain disorder. Int Phys Med Rehab J. 20I8;3(4):279-285.

DOI: 10.15406/ipmrj.2018.03.00116 
analysis for the Global Burden of Disease Study 2013. The Lancet 2015;386(9995):743-800.

2. Fejer R, Kyvik KO, Hartvigsen J. The prevalence of neck pain in the world population: a systematic critical review of the literature. Eur spine J. 2006;15(6):834-848.

3. Hoy D, Brooks P, Blyth F, et al. The epidemiology of low back pain. Best Pract Res Clin Rheumatol. 2010;24(6):769-781.

4. Hakala P, Rimpela A, Salminen JJ, et al. Back, neck, and shoulder pain in Finnish adolescents: national cross sectional surveys. $\mathrm{Br} \mathrm{Med} J$. 2002;325(7367):743.

5. Ferrari R, Russell AS. Regional musculoskeletal conditions: neck pain. Best Pract Res Clin Rheumatol. 2003;17(1):57-70.

6. Cote P, Cassidy JD, Carroll LJ, et al. The annual incidence and course of neck pain in the general population: a population-based cohort study. Pain. 2004;112(3):267-273.

7. McWilliams LA, Cox BJ, Enns MW (2003). Mood and anxiety disorders associated with chronic pain: an examination in a nationally representative sample. Pain. 106(1-2):127-133.

8. Burton AK, Tillotson KM, Main CJ, et al. Psychosocial predictors of outcome in acute and sub chronic low back trouble. Spine. 1995;20(6):722-728.

9. Manchikanti L, Singh V, Datta S, et al. Comprehensive review of epidemiology, scope, and impact of spinal pain. Pain Ph. 2009; 12(4):E35-70.

10. Wiech K, Tracey I. The influence of negative emotions on pain: behavioral effects and neural mechanisms. Neuroimage. 2009; 47(3):987-994.

11. Carosella AM., Lackner JM, Feuerstein. Factors associated with early discharge from a multidisciplinary work rehabilitation program for chronic low back pain. Pain. 1994;57(1):69-76.
12. Fautrel B, Guillemin F. Cost of illness studies in rheumatic diseases. Curr Opin Rheumatol. 2002;14(2):121-126.

13. Linton SJ, Nordin E. A 5-year follow-up evaluation of the health and economic consequences of an early cognitive behavioral intervention for back pain: a randomized, controlled trial. Spine. 2006;31(8):853-858.

14. Nachemson A. Back pain: delimiting the problem in the next millennium. Int J Law Psychiatry. 1999;22(5-6):473-490.

15. Zusman M. Forebrain-mediated sensitization of central pain pathways: 'non-specific 'pain and a new image for MT. Man Ther. 2002;7(2):80-88.

16. Frison L, Pocock SJ. Repeated measures in clinical trials: analysis using mean summary statistics and its implications for design. Stat Med. 1992;11(13):1685-1704.

17. Singh V, Rana RK, Singhal R. Analysis of repeated measurement data in the clinical trials. J Ayurveda Integr Med. 2013;4(2):77 -81.

18. Van Tulder MW, Scholten RJ, Koes BW, et al. Non-steroidal antiinflammatory drugs for low back pain. Cochrane Database Syst Rev. 2000 .

19. Maier W, Buller R, Philipp M, et al. The Hamilton Anxiety Scale: reliability, validity and sensitivity to change in anxiety and depressive disorders. J Affect Disord. 1988;14(1):61-68.

20. Taimela S, Diederich C, Hubsch M, et al. The role of physical exercise and inactivity in pain recurrence and absenteeism from work after active outpatient rehabilitation for recurrent or chronic low back pain: a followup study. Spine. 2000;25(14):1809-1816.

21. Rainville J, Sobel JB, Hartigan C, et al. The effect of compensation involvement on the reporting of pain and disability by patients referred for rehabilitation of chronic low back pain. Spine. 1997;22(17):20162024. 